\title{
The Flyby Anomaly and the Gravitational-Magnetic Field Induced Frame-Dragging Effect around the Earth
}

\author{
Babur M. Mirza \\ Department of Mathematics, \\ Quaid-i-Azam University, 45320 Islamabad, Pakistan
}

September 19, 2019

\begin{abstract}
The anomalous energy difference observed during the Earth flybys is modelled here as a dynamical effect resulting from the coupling of the gravitational and the magnetic fields of the Earth. The theoretical analysis shows that general relativistic frame-dragging can become modified under the Earth's magnetic field by orders of magnitude. For twelve flyby cases, including the null results reported in some recent flybys, the predicted velocities correspond to the observed velocities within the observational error. The gravito-magnetic effect is also shown to account for the linear distance relation, time-variation of the anomalous energy, and the reduction in the anomalous velocity for high altitude flybys near the Earth.
\end{abstract}

\section{Introduction}

Anomalous velocity increase in the flyby orbits has been detected during the Earth flybys of various spacecraft (Anderson 2008; Anderson 2007; Iorio 2009; McCulloch 2008; Nottale 2003). The energy change in the reported flybys corresponds to $10^{-6}$ orders of magnitude on close encounters. The source of the energy anomaly is not known, and it is now well established that the effect cannot be due to systematic errors, nor due to dynamical effects, such as the Earth's rotation, or due to the general relativistic Lense-Thirring frame-dragging of the spacetime. Studies of various theoretical as well as phenomenological models has been made by Lämmerzahl et. al. (Lämmerzahl 2018), including the effects due to the atmosphere, ocean tides, solar wind, and spin-rotation coupling, among others. The general conclusion of these studies is that these perturbations do not correspond to the flyby anomaly, where as in phenomenological models it is necessary to pin down a specific interaction term. 
In general the flyby anomaly has various features related to the velocity increase. It corresponds to a constant acceleration. In some flybys energy decrease has been observed where as in others the energy increases. However, these relate to the signs and magnitudes of the spacecraft's initial and final velocity orientations with respect to the equatorial plane. The empirical formula of Anderson et.al. (Anderson 2008) gives the energy $K$ corresponding to the radius $R_{\oplus}$ of the Earth as $K=2 \omega_{\oplus} R_{\oplus} / c$, where $\omega_{\oplus}$ is the angular rotational speed of the Earth, and $c$ is the speed of light. This formula gives the correct order of magnitude for the orbital energy anomaly, and fits well with the observed change in the orbital parameters due to the change in the velocity. Further studies by Anderson et.al. (Anderson 2007) have shown that the energy transfer during planetary orbital motion occurs as a function of time, which rather than being a monotonic function of time exhibits local maxima and minima. Some recent high altitude flybys have detected a null anomalous effect, which is at variance with the empirical formula $K=2 \omega_{\oplus} R_{\oplus} / c$.

In this paper, it is shown that these features, as well as others related to the flyby anomaly, can be due to the coupling of gravitational and magnetic fields around the Earth. It has been suggested that since the anomalous energy change depends on the latitude, Earth's rotation may be generating an effect much larger than the general relativistic Lense-Thirring frame dragging around the Earth. We show here that the precise nature of this effect, however, is not the Lense-Thirring frame-dragging, but a general relativistic coupling of the magnetic and gravitational fields. Such a coupling can be studied in the context of Maxwell field equations in the linearized Kerr spacetime, where the rotation of the source mass induces the dragging of the background spacetime. In this case the toroidal magnetic field energy density directly modifies the spacetime frame dragging around a source mass, such as the Earth. The estimated dimensionless energy $K$ corresponds to the magnetic field and the radius of the gravitational source, as in the Anderson et. al. formula. However, the magnetic field intensity reduction with altitude reduces the magnetic field energy density, hence the dragging effect at large distances from the Earth. The later case corresponds to the null effect measured by some recent flybys (Jouannic 2015). Estimate of the anomalous velocity for the various flybys are shown to be in agreement with the observed velocity change within the observational errors, as well as the reported null results of the recent flybys. Throughout we use the standard spacetime coordinates $(t, r, \theta, \varphi)$.

\section{General Relativistic Toroidal Field Energy- Density}

In a stable equilibrium configuration, the exterior spacetime of a rotating object of mass $M$ is essentially stationary and axially symmetric. For such a case the spacetime around the gravitational source can be modelled by the linearized 
Kerr metric (Rindler 2006),

$$
g_{\alpha \beta}=\left[\begin{array}{cccc}
-e^{2 \Phi(r)} & 0 & 0 & -\omega(r) r^{2} \sin ^{2} \theta \\
0 & e^{-2 \Phi(r)} & 0 & 0 \\
0 & 0 & r^{2} & 0 \\
-\omega(r) r^{2} \sin ^{2} \theta & 0 & 0 & r^{2} \sin ^{2} \theta
\end{array}\right],
$$

where

$$
e^{2 \Phi(r)}=\left(1-\frac{2 M}{r}\right),
$$

$\omega(r)$ is the Lense-Thirring frame-dragging frequency, equal to $2 \mathcal{J} / r^{3}$, and $\mathcal{J}$ is the angular momentum of the gravitational source (Glendenning 1997). The coupling of the gravitational field to the magnetic field of such an object can be studied using the general relativistic form of the Maxwell field equations in curved spacetimes (Landau \& Lifshitz 1980),

$$
\begin{gathered}
F_{\alpha \beta, \gamma}+F_{\beta \gamma, \alpha}+F_{\gamma \alpha, \beta}=0, \\
\left(\sqrt{-g} F^{\alpha \beta}\right)_{, \beta}=4 \pi \sqrt{-g} J^{\alpha},
\end{gathered}
$$

where $g$ is the determinant of the metric tensor $g_{\alpha \beta}$ given by equation (1), and $F_{\alpha \beta}$ is the electromagnetic field tensor. It can be shown (Lichnerowicz 1967) that the assumption of an everywhere finite current 4-vector $J^{\alpha}$ leads to the condition that in a co-moving frame the electric field 4-vector $E^{\alpha}=0=E_{\alpha}$. Therefore, for the region exterior to the source, the electromagnetic field tensor has the form,

$$
F_{\alpha \beta}=\sqrt{-g} \epsilon_{\alpha \beta \gamma \delta} u^{\gamma} B^{\delta},
$$

and in contravariant form

$$
F^{\alpha \beta}=-\frac{1}{\sqrt{-g}} \epsilon^{\alpha \beta \gamma \delta} u_{\gamma} B_{\delta},
$$

where $\epsilon_{\alpha \beta \gamma \delta}$ is the four index Levi -Civita symbol. Here a convenient and physically meaningful choice of a co-moving observer is the one belonging to the class of observers ZAMOs, having zero angular momentum. This is an observer circling the gravitational source with angular speed $\omega$ at a fixed radial distance $r$, per unit length, and polar angle $\theta$, being brought into motion by dragging of the background spacetime (Rindler 2006). By definition, for a ZAMO $u_{r}$ and $u_{\theta}$ vanish, and the velocity four vector compatible with the metric is given by

$$
u^{\alpha}=\left(e^{-\Phi(r)}, 0,0, \omega e^{-\Phi(r)}\right), \quad u_{\alpha}=\left(-e^{\Phi(r)}, 0,0,0\right) .
$$

Given the current 4 -vector $J^{\alpha}$, the metric tensor $g_{\alpha \beta}$, and the velocity 4 -vector $u^{\alpha}$, the set of equations (3) and (4) can be solved to give the magnetic field as a function of $(t, r, \theta, \varphi)$. The full set of Maxwell equations (3) and (4) gives a system of eight coupled partial differential equations. Solutions to the Maxwell equations in axially symmetric spacetimes has been found (Mirza 2017; Pétri 
2013; Mirza 2007; Herrera et al. 2006; Oron 2002) which satisfy the asymptotic boundary conditions of finite energy density, and other physical conditions such as a bounded magnetic field outside the event horizon. Using equations (5) and (6) in equations (3) and (4), it is easily seen that for $\omega \neq 0$ and $B_{t} \neq 0$, the magnetic field around the star has the form,

$$
\left(B^{\alpha}\right)=B_{0}(0,0, A(\theta) \sin \theta \sin \xi, \cos \xi) /\left(u_{t} r^{2} \sin ^{2} \theta\right)
$$

where $B_{0}$ is the magnetic field intensity constant, $\xi=\varphi-\omega t$ is the travelling wave variable, and $A(\theta)=\int(1 / \sin \theta) d \theta$. Notice that the magnetic field $(8)$ is due to the frame-dragging effect of the background spacetime which vanishes, otherwise, for $\omega=0$. For a free-falling observer, such as the ZAMO, $\omega=d \varphi / d t$, implies that $\xi=\xi_{0}=0$, and the non-vanishing component of the magnetic field is the toroidal component. This is also because the integral $A(\theta)=\int(1 / \sin \theta) d \theta$ is equal to zero for a closed loop around the source. The dragging effect is maximum in the equatorial plane $\theta=\pi / 2$ of the gravitational source, therefore using $B_{T}=\sqrt{B^{\alpha} B_{\alpha}}$, the magnetic field component effective in the equatorial plane of the Earth is the toroidal field component,

$$
B_{T}=-\frac{B_{0}}{r \sqrt{1-\frac{R_{s}}{r}}} \cos (\varphi-\omega t), r>R_{s},
$$

where $R_{s}=2 G M / c^{2}$ is the Schwarzschild radius for the source mass $M$. Also from equations $(2)$ and $(7)$, we have $u_{t}=-\sqrt{1-R_{s} / r}$. For a slowly rotating gravitational source like the Earth $\omega<<1$, and the cosine factor depends on the choice of the coordinate $\varphi$. For the maximum dragging effect we take $\varphi=0$. The radial drag therefore corresponds to $B_{0} /\left(r \sqrt{1-R_{s} / r}\right)$ in the equatorial plane of the rotating gravitational object.

The total energy density in the toroidal field is given by (Landau \& Lifshitz 1971),

$$
\epsilon_{B}=\int_{0}^{R} 4 \pi r^{2} B_{T}^{2} d r
$$

where $R$ is the radius of the source mass. To calculate the energy density (10), we notice that the toroidal field has a discontinuity at the Schwarzschild radius $r=R_{s}$. We thus divide the region of integration in equation (10) as the interior region $r \leq R_{s}$ and the exterior region $r>R_{s}$, such that,

$$
\epsilon_{B}=\int_{0}^{R_{s}} 4 \pi r^{2} B_{T}^{2} d r+\int_{R_{s}}^{R} 4 \pi r^{2} B_{T}^{2} d r
$$

The first integral can be calculated using $B_{T}=B_{0} / r \sqrt{1-R_{s} / r}$, we therefore obtain,

$$
\int_{0}^{R_{s}} 4 \pi r^{2} B_{T}^{2} d r=4 \pi B_{0}\left(R_{s}+R_{s} L t_{\Delta r \rightarrow 0} \ln \Delta r-R_{s} \ln \left|R_{s}\right|\right)
$$


where $\Delta r=\left|r-R_{s}\right|$. Similarly, for the second integral, we have,

$$
\int_{R_{s}}^{R} 4 \pi r^{2} B_{T}^{2} d r=4 \pi B_{0}\left(R-R_{s}+R_{s} \ln \left|R-R_{s}\right|-R_{s} L t_{\Delta r \rightarrow 0} \ln \Delta r\right) .
$$

Putting from (12) and (13) into equation (11), we have for for the exterior region $R>R_{s}$,

$$
\epsilon_{B}=4 \pi B_{0}^{2}\left[R+R_{s} \ln \left(R-R_{s}\right)\right] .
$$

This energy density, although due to the coupling of the magnetic to the gravitational field, contributes to the total energy density around the source mass. Notice that the gravitational field energy depends on the Schwrazschild factor $R_{s}=2 G M / c^{2}$. Since for the Earth, $R>R_{s}$, therefore the second term in equation (14) can be dropped, and the formula for the dimensionless anomalous energy per unit energy density is,

$$
K=4 \pi B_{0}^{2} R,
$$

for $R>R_{s}$.

Some key features of formula (15) are noticeable. (1) The anomalous energy is linearly dependent on the radius of the Earth, as in Anderson et. al. (2008). We thus identify $2 \omega / c$ as the magnetic energy density $4 \pi B_{0}^{2}$, which is also dimensionally consistent by equation (15). (2) When the test body moves towards the source, the total energy density will be reduced, since the work done is opposite to the force field, where as contrary will be the case for an outward motion. (3) For a time-varying field, the anomalous energy should exhibit an oscillatory trend with time as $\cos ^{2}(\varphi-\omega t)$, as reported in Anderson et.al. (2007).

The energy given by equation (15) is the kinetic energy per unit normalized energy density $E$ in the equatorial plane of the Earth. The effective components along the velocity vectors in the plane of the orbit are $4 \pi B_{0}^{2} R \cos \phi_{i}$ and $4 \pi B_{0}^{2} R \cos \phi_{o}$, where $\phi_{i}$ and $\phi_{o}$ are the incoming and outgoing orbital latitudes of the flyby, respectively. Also, since for the velocity change $d V / V_{\infty}=d E / 2 E=$ $K\left(\cos \phi_{i}-\cos \phi_{o}\right)$, we have,

$$
d V=4 \pi B_{0}^{2} R V_{\infty}\left(\cos \phi_{i}-\cos \phi_{o}\right)
$$

where $V_{\infty}$ is the asymptotic (hyperbolic) excess velocity of the flyby. The net velocity change is twice the velocity increase (or decrease) along the incoming or outgoing trajectories. The average surface magnetic field of the Earth approximately varies from $0.3 G$ to $0.6 G$. We take the surface magnetic field in calculations (Table 1 ) to be $0.5 G$ in cgs units. The corresponding magnetic field in SI units is of the order $0.0126 \times 0.5 A$ per unit distance. According to equation (15), the magnetic field weakens as $1 / \sqrt{h}$ where $h$ is the height of the test particle above the Earth's surface. For flyby encounters the average distance of $10^{3} \mathrm{~km}$ implies a reduction in the magnetic field by an order $10^{-3}$.

In Table 1 , the estimated velocity change $d V_{i}$, using equation (16), is compared with the observed velocity change $d V_{o}$ for flybys of twelve spacecraft. 
In the case of last six flybys the average asymptotic speed is taken to be the average speed of $10 \mathrm{~km} / \mathrm{s}$. The minimum altitude is taken to be the height $h$ above the Earth surface, measured during close encounters. In relation (15) the intrinsic magnetic field $B_{0}$ and the radius $R$ of the gravitating body are the only input parameters needed to estimate the anomalous energy. For the Earth, the estimated dimensionless anomalous energy $K$ comes out to be about $3.1 \times 10^{-6}$, which is comparable with the observed average energy difference for the flybys. For planets with intrinsic dipolar magnetic fields of similar orders of magnitude, but larger radii, the estimated anomalous energy near the surface also has a comparable magnitude for $K$.

\begin{tabular}{|l|l|l|l|l|l|l|l|}
\hline Mission & $V_{\infty}(\mathrm{km} / \mathrm{s})$ & $h(\mathrm{~km})$ & $\phi_{i}$ & $\phi_{o}$ & $\Delta$ & $d V_{o}(\mathrm{~mm} / \mathrm{s})$ & $d V_{p}(\mathrm{~mm} / \mathrm{s})$ \\
\hline Galileo-I & 8.949 & 960 & -12.52 & -34.15 & 0.149 & $3.92 \pm 0.3$ & 4.133 \\
\hline Galileo-II & 8.877 & 303 & -34.26 & -4.87 & -0.17 & $-4.6 \pm 1.0$ & -4.678 \\
\hline NEAR & 6.851 & 539 & -20.76 & -71.96 & 0.626 & $13.46 \pm 0.01$ & 13.295 \\
\hline Cassini & 16.010 & 1175 & -12.92 & -4.99 & -0.022 & $-2 \pm 1$ & -1.09 \\
\hline Rosetta & 3.863 & 1955 & -2.81 & -34.29 & 0.172 & $1.80 \pm 0.03$ & 2.058 \\
\hline Messenger & 4.056 & 2347 & 31.44 & -31.92 & $5 \times 10^{-3}$ & $0.02 \pm 0.01$ & 0.062 \\
\hline Rosetta-II & 10.0 & 5301 & -10.80 & 18.60 & 0.034 & 0 & 0.1 \\
\hline EPOXY-I & 10.0 & 15614 & -5.18 & 16.90 & 0.039 & 0 & 0.12 \\
\hline EPOXY-II & 10.0 & 43415 & 17.03 & 64.12 & 0.519 & 0 & 1.6 \\
\hline Rosetta-III & 10.0 & 2480 & 18.49 & 24.37 & 0.040 & 0 & 0.12 \\
\hline EPOXY-III & 10.0 & 30404 & -63.88 & -8.04 & 0.549 & 0 & 1.7 \\
\hline Juno & 10.0 & 561 & 14.17 & 39.50 & 0.197 & 0 & 0.6 \\
\hline
\end{tabular}

Table I: The anomalous change in the flyby velocity as predicted $\left(d V_{p}\right)$ from equation (16), and compared with the observed $\left(d V_{o}\right)$ velocity change for flyby missions during 1990-2013. The asymptotic velocity $V_{\infty}$ is measured in $\mathrm{km} / \mathrm{s}$ (Anderson et. al. 2008; Jouannic et. al. 2015), and $\Delta=\cos \phi_{i}-\cos \phi_{o}$. The scaled dimensionless radius $R_{\oplus}$ for the Earth is in per unit length of $k m$, where as the average surface magnetic field of the Earth is $0.5 G$ over unit distance per $k m$.

\section{Conclusions and Discussion}

In Table 1, data (Anderson et.al. 2008; Jouannic et. al. 2015) of six flyby missions, including the observed and calculated velocity changes, is given. The anomalous velocity $d V$ exhibits positive and negative signs, and in the case of Messenger flyby the anomalous effect is close to zero. We notice that the dragging effect is maximum in the equatorial plane of the Earth and decreases towards the poles. Resultingly, for a small ingoing declination angle $\phi_{i}$ the dragging effect is larger as compared to a larger outgoing declination angle. The velocity change is therefore positive as in the case of Galileo-I, NEAR, and Rosetta flybys. Also, since the velocity change depends on the flyby asymptotic velocity $V_{\infty}$ relative to the Earth, the spin rotation of the Earth determines the sign of the anomalous velocity change. For the case of Messenger the ingoing and outcoming declination angles are approximately equal, hence the drag along 
the orbital motion towards and away from the source, approximately cancels. In this case the net velocity change is small.

We recover the Anderson et.al. empirical formula $K=2 \omega_{\oplus} R_{\oplus} / c$ by observing that near the Earth's surface change in the energy density due to the spacetime dragging is proportional to the change in the rotational velocity of a particle placed close to the surface of the Earth. Therefore, in one cycle, $\omega$ varies as $2 \pi B_{0}^{2}$. With speed of light $c$ being the constant of proportionality, this yields the relation $K=2 \omega R / c$. However, in the empirical formula of Anderson et. al. the effects of rotation do not depend on the flyby's distance from the Earth. We observe that for the dimensionless energy $K$, equation (16) implies that $B_{0}$ is reduced as $1 / \sqrt{h}$, where $h$ is the height of the test particle above the Earth's surface. The magnetic field intensity thus decreases by a factor $10^{-3}$ units at an altitude of $10^{6} \mathrm{~m}$, which is the approximate distance of the flybys in Table 1. In the high altitude flyby encounters, such as in the case of Rosetta-II, EPOXY-II, and EPOXY-III, the average distance of the flyby in trajectory is approximately 10 - 50 times the flyby altitudes reported in Anderson et.al. The resulting anomalous velocity change therefore diminishes as $B_{0}^{2} \sim 10^{-1}-2 \times 10^{-2}$ units, hence tends to lie below the range of the observational error in high altitude flybys. As reported in Jouannic et.al., no anomalous velocity change was observed for Rosetta-II, and for EPOXY-II and III flybys. In closed orbital motion, however, the energy density have the cumulative effect over time. Thus for the lunar orbit, net velocity drag along the radial direction is $\sqrt{4 \pi R} B_{0} \approx 10^{-9} \mathrm{~m} / \mathrm{s}$ per year. This is comparable with the observed lunar orbital recession speed (Anderson \& Nieta 2009).

The coupling of gravitational field to the magnetic field in extreme gravitomagnetic environments is well-studied, and leads to an enhancement of the magnetic field energy density of a star. This however contributes to the total energy density of the field around a massive object, possessing both magnetic field and rotation. In neutron stars and magnatars the wrapping of the spacetime around the star can enhance the energy density up to several orders of magnitude (Mirza 2017). Analogous conditions exist in almost all gravitationally bound systems with a dipolar magnetic field and a toroidal field component. Although, in such cases, the magnetic field is weak and rotation is slow, the coupling effect can possess measurable magnitude. The effect derived here is due to rotation but cannot strictly be the Lense-Thiring frame-dragging, where only the gravitational field is involved, and dragging occurs close to the surface of the star. Magnetic field causes a more effective dragging of the spacetime, thus enhances the spacetime frame-dragging up to comparatively larger distances.

\section{References}

[1] Anderson, J. D., Campbell, J. K., and Nieto, M. M., 2007, New Astron. Rev. 12, 383. 
[2] Anderson, J. D., Campbell, J. K., Ekelund, J. E., Ellis, J., and Jordan, J. F., 2008, PRL 100, 091102.

[3] Anderson, J. D., and Nieto, M. M., 2007, arXiv:0907.2469.

[4] Antreasian, P. G., Guinn, J. R., 1998, Astrodynamics Specialist Conference (AIAA, Washington), pp. 98-4287.

[5] Glendenning, N.K., 1997, Compact Stars, (Springer-Verlag, New York), pp. 254-255.

[6] Herrera, L., González, G. A., Pachón, L. A., \& Rueda, J. A., 2006, Class. Quantum Grav. 23, 2395.

[7] Iorio, L., 2009, Scholarly Research Exchange 2009.

[8] Jouannic, B., Noomen, R., and van den IJssel, 2015, JAA. Proceedings of the international symposium on space flight dynamics, ed. R. Kahle (DLR, Munich), pp. 1-17.

[9] Lämmerzahl, C., Preuss, O., \& Dittus, H., 2018, arXiv:gr-qc/0604052.

[10] Landau, L. D. \& E.M. Lifshitz, E. M.,1971, The Classical Theory of Fields, (Pergamon, New York), p. 67-74.

[11] McCulloch, M. E., 2008, MNRAS Letters, 389, L57.

[12] Mirza, B.M., 2007, Int. J. Mod. Phys. D16 1705 (2007).

[13] Mirza, B.M. 2017, ApJ. 847, 73.

[14] Nottale, L., 2003, arXiv preprint gr-qc/0307042.

[15] Oron, A., 2002, Physical Review D66, 023006.

[16] Pétri, J., 2013, MNRAS 433, 986.

[17] Rindler, W., 2006, Relativity: Special, General, and Cosmological, second edition, (Oxford University Press, Oxford), pp.337-339. 